\title{
Review Article \\ Diverse Features of the Multiwavelength Afterglows of Gamma-Ray Bursts: Natural or Special?
}

\author{
J. J. Geng ${ }^{1,2}$ and Y. F. Huang ${ }^{1,2}$ \\ ${ }^{1}$ School of Astronomy and Space Science, Nanjing University, Nanjing 210046, China \\ ${ }^{2}$ Key Laboratory of Modern Astronomy and Astrophysics (Nanjing University), Ministry of Education, Nanjing, China \\ Correspondence should be addressed to Y. F. Huang; hyf@nju.edu.cn
}

Received 22 February 2016; Revised 7 April 2016; Accepted 10 April 2016

Academic Editor: WeiKang Zheng

Copyright (c) 2016 J. J. Geng and Y. F. Huang. This is an open access article distributed under the Creative Commons Attribution License, which permits unrestricted use, distribution, and reproduction in any medium, provided the original work is properly cited.

The detection of optical rebrightenings and X-ray plateaus in the afterglows of gamma-ray bursts (GRBs) challenges the generic external shock model. Recently, we have developed a numerical method to calculate the dynamics of the system consisting of a forward shock and a reverse shock. Here, we briefly review the applications of this method in the afterglow theory. By relating these diverse features to the central engines of GRBs, we find that the steep optical rebrightenings would be caused by the fall-back accretion of black holes, while the shallow optical rebrightenings are the consequence of the injection of the electron-positron-pair wind from the central magnetar. These studies provide useful ways to probe the characteristics of GRB central engines.

\section{Introduction}

It is believed that gamma-ray bursts (GRBs) are generated from either the collapse of massive stars [1-4] or the merger of neutron stars (NSs) [5-7], during which collimated relativistic outflows can be launched. As the outflow propagates into the circum-burst medium, a relativistic blast wave will develop, whose dynamic evolution can be well described by the Blandford-McKee solution [8]. The blast wave would sweep up and accelerate the circum-burst electrons and generate afterglows at frequencies ranging from $\mathrm{X}$-rays to radio waves [9-11]. This is the basic picture for GRB afterglows. In the pre-Swift era, many afterglow lightcurves showed a smooth power-law decay, which can be explained by the synchrotron radiation from electrons accelerated by the forward shock (FS). For a complete reference of the analytical synchrotron external shock afterglow models, one can see [12]. However, many unexpected features in the afterglows were later observed thanks to the Swift satellite [13-15] and other optical telescopes (e.g., GROND telescope; see [16]).

Early flares and shallow decay phase (or the so-called Xray plateau) are common in the X-ray afterglow data [17]. This indicates that the central engines of GRBs are still active after the burst, giving us a useful clue to investigate the central engines. On the other hand, some optical afterglows show rebrightenings at late stages in the observer frame $\left(t_{\mathrm{obs}} \sim 10^{4}-10^{5} \mathrm{~s}\right)$. In some cases, bumps in X-rays are accompanied by optical rebrightenings (e.g., GRB 120326A $[18,19])$, while, in other cases, no clear counterpart features are observed in optical bands (e.g., GRB 100814A [20]). Both the X-ray plateaus and the optical rebrightenings cannot be explained in the framework of a simple FS scenario. Thus researchers have proposed several refined models to interpret these unexpected features in recent years (see [21, 22] for a review). Stimulated by these refined models, it is urgent to answer whether X-ray plateaus and optical rebrightenings have a natural origin or they are special outcomes varying in different GRBs.

Normally, the energy released during the X-ray plateau is several percent of the prompt emission [23], which motivates researchers to favour the scenarios involving energy injection processes. According to the composition of the injected late outflow, there are generally three types of energy injection processes, that is, the pure Poynting-flux injections [24-27], the collision of kinetic energy dominated shells [28], and the injection of the electron-positron-pair winds $\left(e^{+} e^{-}\right.$winds $[29,30])$. If one further considers the optical rebrightenings, 
some other scenarios are called for, including the circumburst density jumps [31-33], two-component jets [34, 35], and varying microphysical parameters [36]. All these models have succeeded to some extent in explaining one or several afterglows according to previous studies. On the other hand, most researchers believe that the central engines of GRBs are either black holes (BHs) or magnetars. Therefore, it may be reasonable to deduce that some specific groups of afterglows should have common features, and these features are associated with the physics of the central engines.

According to previous researches, late activities of $\mathrm{BHs}$ may be sustained by the accretion of fall-back material that fails to escape from the progenitor star [37-39]. The energy injection is expected to be delayed by the fall-back time $t_{\mathrm{fb}}$. If the FS is affected by such a delayed energy injection, the shock dynamics should rapidly evolve from a noninjection phase to an injection-dominated phase [40]. As a result, afterglows with steep optical rebrightenings (with the time scale of the rebrightening $\delta t_{\text {obs }}<t_{\text {obs }}$ ) are generated. We thus proposed that steep optical rebrightenings are caused by the fall-back processes of central accreting BHs. By contrast, the energy flow from a magnetar may be in the form of a continuous $e^{+} e^{-}$wind. The $e^{+} e^{-}$wind model was initially proposed to explain the X-ray plateau [41]. The end time of the plateau phase is roughly the typical spin-down timescale $\left(T_{\text {sd }}\right)$ of the newly born magnetar. For the broad and shallow optical rebrightening, its peak time also coincides with $T_{\text {sd }}$, which motivates us to believe that the $e^{+} e^{-}$wind model should work for afterglows with both a shallow optical rebrightening and an accompanied X-ray feature.

We have developed a semianalytic method to solve the dynamic of a system including a FS and a reverse shock (RS). It can be applied in different situations such as when a density jump medium or the $e^{+} e^{-}$wind is involved. In this review, we briefly describe our related studies in recent years and show how the investigations help to shed light on the nature of GRBs. In Section 2, we revisit the circum-burst density jump scenario and compare our results with previous hydrodynamic simulations $[42,43]$. The delayed energy injection model is discussed in Section 3. In Section 4, we show that the $e^{+} e^{-}$wind model would naturally produce the optical rebrightenings and some characteristics of the central magnetar may be derived by comparing the theoretical results with observations. Finally, our conclusions are summarized in Section 5.

\section{Density Jump Scenario}

After the prompt emission of GRB, a FS will form and propagate into the circum-burst medium. The dynamic of the FS can be described by a set of differential equations proposed by [44-46]. Assuming the number density profile of the circum-burst medium is a step function of radius $R$, $n=n_{0}\left(R<R_{0}\right)$ and $n=n_{1}\left(R \geq R_{0}\right)$, where $R_{0}$ is the transition radius and $n_{1}>n_{0}$. Before the FS reaching $R_{0}$, the evolution of the Lorentz factor of the FS $\left(\Gamma_{2}\right)$ is given by [47]

$$
\frac{d \Gamma_{2}}{d m_{2}}=-\frac{4\left(\Gamma_{2}^{2}-1\right)}{8\left(1-\varepsilon_{2}\right) \Gamma_{2} m_{2}+3 \varepsilon_{2} m_{2}+3 M_{\mathrm{ej}}}
$$

where $M_{\mathrm{ej}}$ is the initial mass of the outflow, $m_{2}$ is the mass of the ambient medium swept up by the FS, and $\varepsilon_{2}$ is the radiation efficiency of the shocked material. The subscript " 2 " is used to mark quantities in the shocked region.

When the FS encounters the density jump at $R_{0}$, a RS will form and propagate back into the hot shell [48, 49]. These two shocks (FS and RS) and the contact discontinuity will separate the system into four regions: (1) unshocked highdensity medium, (2) forward-shocked high-density medium, (3) reverse-shocked hot shell, and (4) unshocked hot shell. In this paper, quantities in Region " $i$ " are denoted by subscripts "i." We extend the derivation of [44] to include the role of the reverse shock. Firstly, it is assumed that the Lorentz factors of Region $2\left(\Gamma_{2}\right)$ and Region $3\left(\Gamma_{3}\right)$ are equal; that is, $\Gamma_{2}=\Gamma_{3}=\Gamma$. Secondly, we can calculate the energy of each region $E_{i}$ and the total energy $E_{\text {tot }}=\sum_{i=2}^{4} E_{i}$. For the mass increment of Region 3,dm $m_{3}$, the radiative energy of the FS-RS system is $d E_{\text {rad }}$. By equating $d E_{\text {tot }}$ with $d E_{\text {rad }}$ and using some additional equations, we can obtain

$$
\frac{d \Gamma_{2}}{d m_{2}}=-\frac{(4 / 3)\left(\Gamma_{2}^{2}-1\right)+f_{1}\left(d m_{3} / d m_{2}\right)+\left(1-\varepsilon_{3}\right) f_{2} \Gamma_{2} \Gamma_{42}\left(1-\beta_{42} / \beta_{4}\right) m_{3}\left(d \psi_{4} / d m_{2}\right)}{(8 / 3)\left(1-\varepsilon_{2}\right) \Gamma_{2} m_{2}+\varepsilon_{2} m_{2}+\left(1-\varepsilon_{3}\right) f_{3} m_{3}+\varepsilon_{3} m_{3}}
$$

where $\Gamma_{42}\left(\beta_{42}\right)$ is the relative Lorentz factor (velocity) of Region 4 as measured in the rest of the frame of Region $2, \beta_{4}$ is the velocity of Region 4 , and $f_{1}, f_{2}, f_{3}$, and $\psi_{4}$ are functions of other variables (see Appendix A of [47]). $\Gamma_{2}\left(R>R_{0}\right)$ could be finally derived with some other equations involving $m_{2}$, $m_{3}, \Gamma_{4}$, and $R$.

Here, we briefly describe how the flux densities are calculated after solving (1) and (2). Customarily, the energy distribution function, $d N_{e}^{\prime} / d \gamma_{e}^{\prime}$, of the shocked electrons is taken as the two-segment power-law form [50], with the indices of $-p$ and $-p-1$. Hereinafter, we use prime $\left({ }^{\prime}\right)$ on variables to denote quantities in the shock comoving frame and characters without a prime to denote quantities in the observer frame. Synchrotron and inverse Compton (IC) radiation are then considered to calculate the emission from electrons. Basic formulation can be found in $[45,51-53]$. Finally, the observed flux densities are obtained by integrating emission from electrons on the equal arrival time surface $[54,55]$.

According to some previous studies [31-33], the optical rebrightening is attributed to the radiation from Region 3. However, results from our refined calculations are different. In our work, we find that the magnitude of the emission from Region 3 is mainly determined by two factors. One is the 
thermal Lorentz factor of baryons in Region 3; that is, $\Gamma_{43}$ $\left(\Gamma_{43}=\Gamma_{42}\right)$. The other factor is the number density of electrons in Region $3, n_{3}^{\prime}$. We set the initial values of the outflow parameters as the isotropic kinetic energy $E_{K \text {,iso }}=10^{53} \mathrm{erg}$, the initial Lorentz factor $\Gamma_{2,0}=300, n_{0}=1 \mathrm{~cm}^{-3}, R_{0}=$ $3.4 \times 10^{17} \mathrm{~cm}$, and the redshift $z=1$. In Figure 1, we calculate two cases with different density jump ratios $\left(n_{1} / n_{0}\right)$, of 10 and 100 times, respectively. Results from our semianalytic method show that $\Gamma_{43}$ given by the analytical solution is overestimated (see Figure 1). Moreover, we use the comoving volume of Region 3 to calculate the volume-averaged $n_{3}^{\prime}$, which is significantly lower than that predicted by the shock jump conditions (see Figure 2 of [47]). As a result, the radiation from Region 3 is actually lower than that given by previous analytical studies. Figure 2 shows the corresponding lightcurves in two cases. No notable bumps emerge after the density jump in these cases. This is consistent with the results from several hydrodynamic simulations [42, 43]. In our calculations, typical values are adopted for parameters of the plasma in all regions [56]; that is, the equipartition parameter for electron energy $\epsilon_{e}=0.1$, the equipartition parameter for magnetic field energy $\epsilon_{B}=0.01$, the electron distribution index $p=2.3$, and the half-opening angle $\theta_{j}=$ 0.1 .

\section{Delayed Energy Injection Model}

We now focus on the afterglows with steep optical rebrightenings. We show that the steep optical rebrightening can be naturally generated in the delayed energy injection scenario. In this model, the central engine after burst is assumed to be a $\mathrm{BH}$. Considering the vicinity along the spin axis of the $\mathrm{BH}$ at late times should be clean, we can assume the energy flow is in type of Poynting-flux. The delayed Poynting-flux would be absorbed by the hot plasma behind the FS and modify the dynamics of the FS. If the luminosity of the Poynting-flux is $L$, then the dynamic of the FS can be described by [40]

$$
\begin{aligned}
& \frac{d \Gamma}{d m} \\
& =-\frac{\left(\Gamma^{2}-1\right)-\left((1-\beta) / \beta c^{3}\right) \Omega_{j} L\left(t_{b}-R / c\right)(d R / d m)}{M_{\mathrm{ej}}+2(1-\varepsilon) \Gamma m+\varepsilon m},
\end{aligned}
$$

where $\Gamma=1 / \sqrt{1-\beta^{2}}$ is the bulk Lorentz factor of the FS, $\Omega_{j}=\left(1-\cos \theta_{j}\right) / 2$ is the beaming factor of the GRB outflow, $m$ is the swept-up mass by the shock, $\varepsilon$ is the radiative efficiency, $R$ is the radius of the FS, and $t_{b}$ is the time from the event measured in the burster frame. Such a delayed energy injection would lead to a rapid change in the evolution of $\Gamma$ according to (3); consequently the flux would show a steep rise.

The energy injection power (with a luminosity of $L$ ) during the fall-back accretion may come from some magnetic processes [58-60]; however, its exact temporal profile is still uncertain. Here, we use two possible modes in our calculations. One is the top-hat mode in which the injected power $L$ is a constant from a start time $t_{\mathrm{obs}}^{\mathrm{s}}$ to an end time $t_{\text {obs }}^{\mathrm{e}}$ [38]. The other is the broken-power-law mode, in which the luminosity profile is similar to the profile of the mass accretion rate during the fall-back $[3,39,61,62]$; that is,

$$
\begin{aligned}
L= & L_{\mathrm{p}}\left[\frac{1}{2}\left(\frac{t_{\mathrm{obs}}-t_{\mathrm{obs}}^{\mathrm{s}}}{t_{\mathrm{obs}}^{\mathrm{p}}-t_{\mathrm{obs}}^{\mathrm{s}}}\right)^{-\alpha_{r} s}\right. \\
& \left.+\frac{1}{2}\left(\frac{t_{\mathrm{obs}}-t_{\mathrm{obs}}^{\mathrm{s}}}{t_{\mathrm{obs}}^{\mathrm{p}}-t_{\mathrm{obs}}^{\mathrm{s}}}\right)^{-\alpha_{d} s}\right]^{-1 / s},
\end{aligned}
$$

where $L_{\mathrm{p}}$ is the peak luminosity at the peak time $t_{\mathrm{obs}}^{\mathrm{p}}, \alpha_{r}$, $\alpha_{d}$ are the rising and decreasing index respectively, and $s$ is the sharpness of the peak. In Figure 3, we show lightcurves calculated in the two modes, from which we find that the steep optical rebrightening would be generated. The initial conditions of the outflow and the key parameters involved in the radiation process are the same as those in Section 2.

This model has been used to interpret the steep rebrightenings in the lightcurves of GRB 081029 [40, 63, 64] and GRB $100621 \mathrm{~A}[40,65]$. We notice that $t_{\mathrm{obs}}^{\mathrm{s}} /(1+z)$ is just equal to $t_{\mathrm{fb}}$, which gives

$$
\frac{t_{\mathrm{obs}}^{\mathrm{s}}}{1+z} \simeq\left(\frac{\pi^{2} r_{\mathrm{fb}}^{3}}{8 G M_{\mathrm{BH}}}\right)^{1 / 2}
$$

where $r_{\mathrm{fb}}$ is the fall-back radius, $G$ is the gravitational constant, and $M_{\mathrm{BH}}$ is the mass of central $\mathrm{BH}$. Moreover, the injected energy should come from the potential energy of the fall-back material; that is,

$$
\frac{\Omega_{j}}{1+z} \int_{t_{\mathrm{obs}}^{\mathrm{s}}}^{t_{\mathrm{obs}}^{\mathrm{e}}} L d t_{\mathrm{obs}} \simeq \eta \frac{G M_{\mathrm{BH}} M_{\mathrm{fb}}}{r_{\mathrm{fb}}},
$$

where $M_{\mathrm{fb}}$ is the total fall-back mass and $\eta$ is the efficiency of the energy conversion. In the top-hat mode, (5) and (6) will give

$$
\begin{aligned}
M_{\mathrm{fb}}= & \frac{2}{1+z} \eta^{-1}\left(\frac{t_{\mathrm{obs}}^{\mathrm{s}}}{1+z}\right)^{2 / 3}\left(\pi G M_{\mathrm{BH}}\right)^{-2 / 3} \\
& \cdot \Omega_{j} L\left(t_{\mathrm{obs}}^{\mathrm{e}}-t_{\mathrm{obs}}^{\mathrm{s}}\right) .
\end{aligned}
$$

Therefore, in principle, $M_{\mathrm{fb}}$ can be inferred from the fitting to the optical rebrightenings in the scenario.

\section{4. $e^{+} e^{-}$Wind Model}

In the prior section, we interpret the steep optical rebrightenings by using the delayed energy injection model. Now, we discuss another group of afterglows, of which the optical rebrightenings are shallower.

After a GRB, the remaining object of the progenitor may be a magnetar, which will lose its rotational energy by ejecting a continuous Poynting-flux. In the $e^{+} e^{-}$wind model, the Poynting-flux may convert into an $e^{+} e^{-}$wind, as hinted from phenomena associated with pulsar wind nebulae [66-68]. As the $e^{+} e^{-}$wind catches up with the FS, a long-lasting RS will form and propagate back into the $e^{+} e^{-}$wind. The RS-shocked 


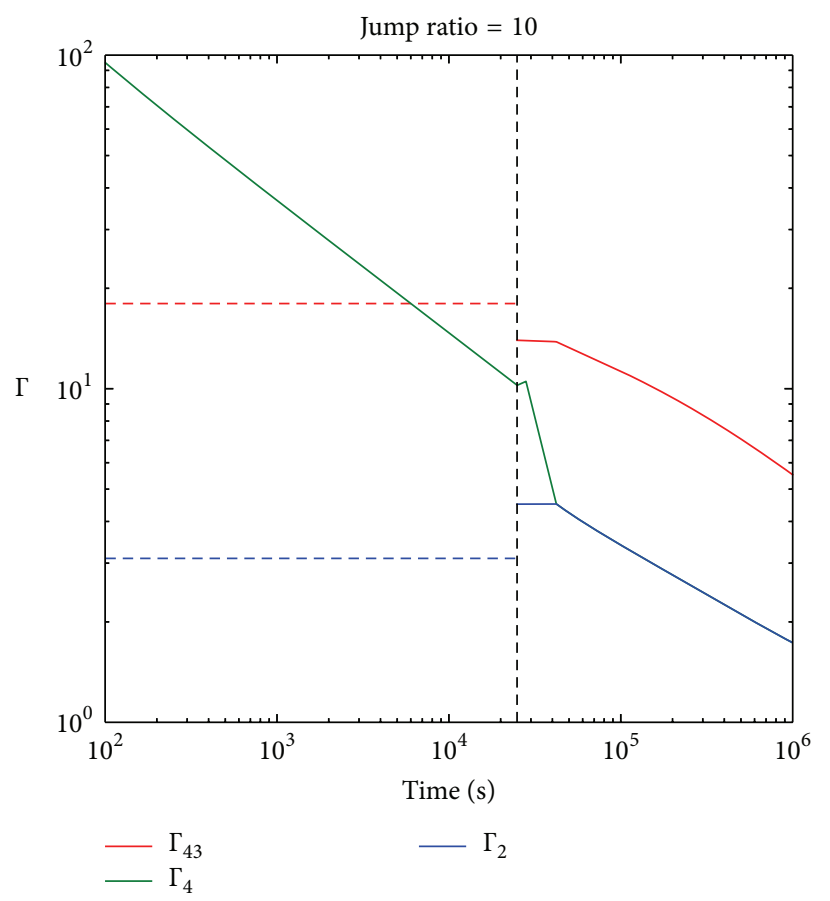

(a)

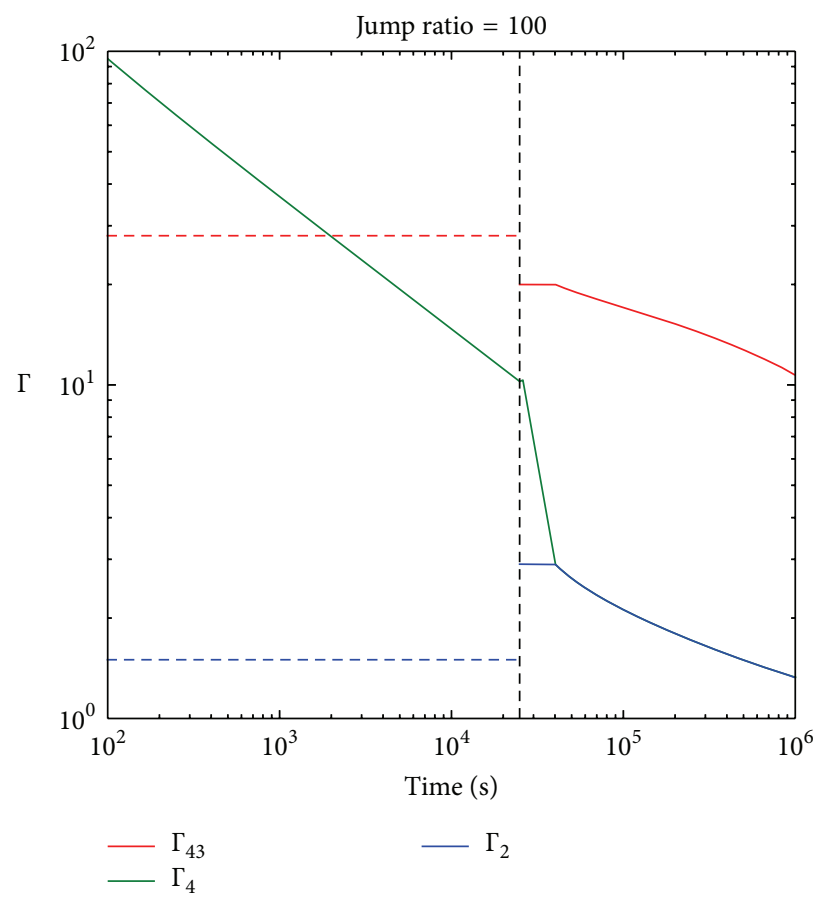

(b)

FIGURE 1: Evolution of the Lorentz factors of different regions when there is a density jump [47]. Two cases are calculated: one corresponds to the density jump ratio of 10 (a), and the other corresponds to the jump ratio 100 (b). The vertical dashed line marks the time of encountering the density jump. The green solid lines represent $\Gamma_{2}$ before encountering the density jump and $\Gamma_{4}$ during the encountering, both denoted by $\Gamma_{4}$. The blue solid lines and red solid lines represent $\Gamma_{2}\left(\right.$ or $\left.\Gamma_{3}, \Gamma_{2}=\Gamma_{3}\right)$ and the thermal Lorentz factor of the baryons in Region $3\left(\Gamma_{43}\right)$ after the encountering, respectively. The horizontal dashed lines mark the values of corresponding Lorentz factors (which remain constant during the reverse shock crossing time) given by [57]. Note that $\Gamma_{4}$ (see the green solid lines) during the encounter is almost constant (slowly increasing due to adiabatic expansion), which is shown as a "plateau" that is significantly shorter than that of $\Gamma_{2}$. The shortness of the "plateau" of $\Gamma_{4}$ is due to the different transformation formula between the burst frame time and the apparent time in the observer frame.

$e^{+} e^{-}$will act as another emitting source besides the electrons shocked by the FS. Consequently, the afterglow lightcurves are the combination of two components. This model has been proposed to interpret the X-ray plateau previously. However, we find this model may account for the common origin for shallow optical rebrightenings around $10^{4} \mathrm{~s}$.

For a newly born magnetar, its Poynting-flux luminosity $L_{w}\left(t_{\mathrm{obs}}\right)$ is [69]

$$
\begin{aligned}
L_{w} \simeq & 4.0 \\
& \times 10^{47} B_{\mathrm{NS}, 14}^{2} R_{\mathrm{NS}, 6}^{6} P_{\mathrm{NS},-3}^{-4}\left(1+\frac{t_{\mathrm{obs}}}{T_{\mathrm{sd}}}\right)^{-2} \mathrm{erg} \mathrm{s}^{-1},
\end{aligned}
$$

and its spin-down timescale is

$$
T_{\mathrm{sd}} \simeq 5.0 \times 10^{4}(1+z) B_{\mathrm{NS}, 14}^{-2} I_{45} R_{\mathrm{NS}, 6}^{-6} P_{\mathrm{NS},-3}^{2} \mathrm{~s},
$$

where $B_{\mathrm{NS}}, R_{\mathrm{NS}}, P_{\mathrm{NS}}$, and $I$ are the surface magnetic field strength, radius, spin period, and moment of inertia of the magnetar, respectively. The convention $Q_{x}=Q / 10^{x}$ in cgs units is adopted hereafter. We assume the Poyntingflux is converted into $e^{+} e^{-}$pairs; then the particle density in the comoving frame of the unshocked wind is $n_{4}^{\prime}=$
$L_{w} /\left(4 \pi R^{2} \Gamma_{4}^{2} m_{e} c^{3}\right)$, where $\Gamma_{4}$ is the bulk Lorentz factor of the unshocked wind (referred to as Region 4 below). Unlike the rapid evolution of $\Gamma_{2}$ in the delayed energy injection scenario, the evolution of $\Gamma_{2}$ is shallow due to the smoothly evolving $L_{w}$ here.

The dynamics of the FS-RS system here can be solved by applying the method mentioned in Section 2. Meanwhile, another method, called the mechanical method [70, 71], was also proposed to solve the dynamics of such FS-RS system. Here, we first compare these two methods. Let us consider an outflow with an isotropic kinetic energy of $E_{K \text {,iso }}=8.0 \times$ $10^{52} \mathrm{erg}$ and an initial Lorentz factor of $\Gamma_{2,0}=150$, and we set $n_{0}=0.1 \mathrm{~cm}^{-3}, \Gamma_{4}=10^{4}$, and $B_{\mathrm{NS}}=2 \times 10^{14} \mathrm{G}$. The evolution of $\Gamma_{2}$ can thus be obtained by using the two methods, respectively. In Figure 4, we see that the results from our method and the mechanical method are consistent with each other. Below, we adopt the mechanical method to solve the dynamics of the FS-RS system.

After taking the parameters $\epsilon_{e, 2}=0.05, \epsilon_{B, 2}=0.01$, $\epsilon_{B, 3}=0.2, \epsilon_{e, 3}=1-\epsilon_{B, 3}=0.8, p_{2}=2.1$, and $p_{3}=2.4$, the corresponding lightcurves can be calculated (see Figure 5). In this case, it is clearly shown that the flux from the RS begins to exceed that from the FS at $\sim 5 \times 10^{4} \mathrm{~s}$, 


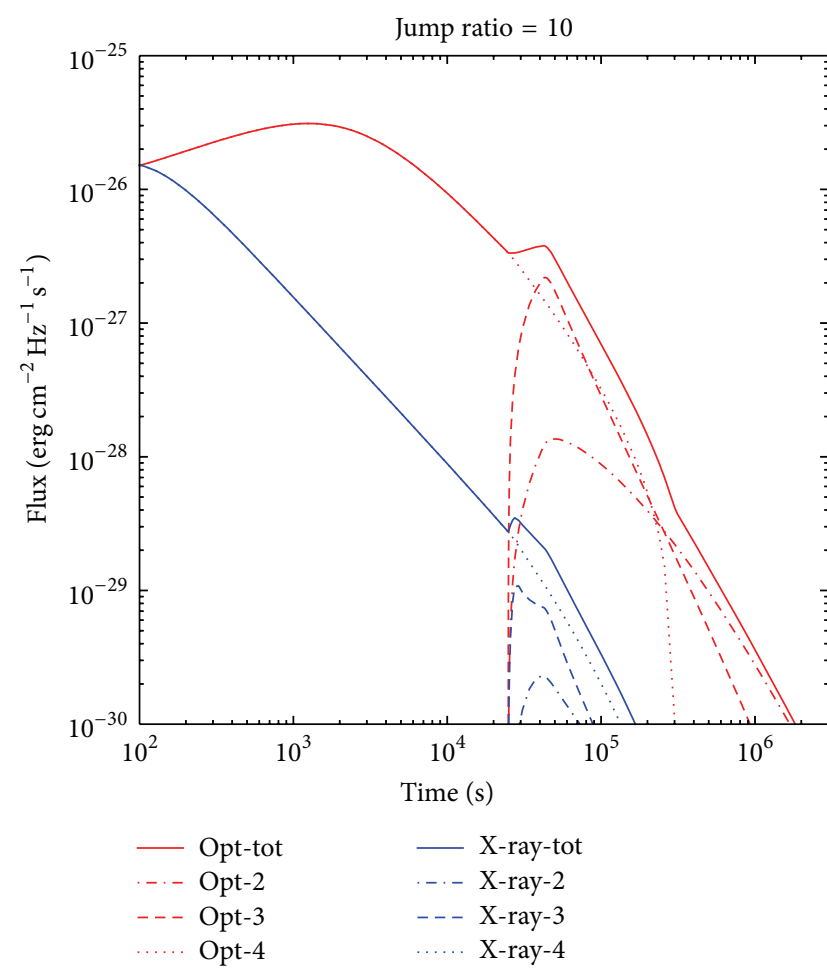

(a)

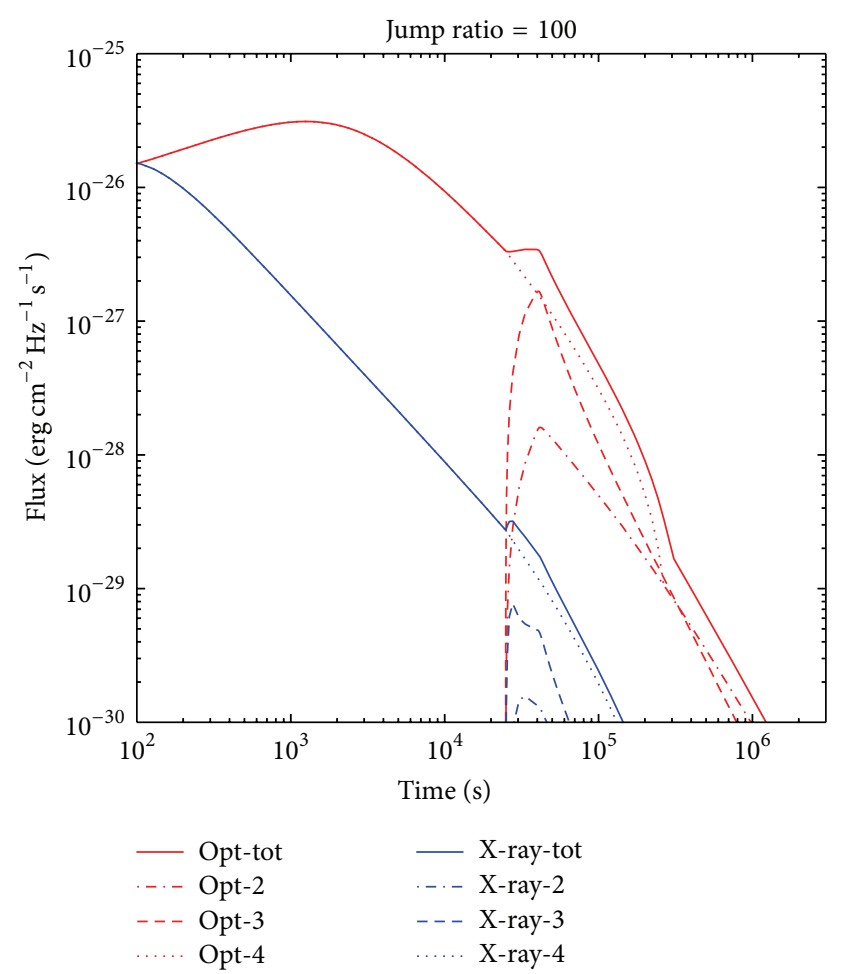

(b)

FIGURE 2: Corresponding afterglow lightcurves for the two cases in Figure 1 . The red, blue lines are lightcurves in the optical band $\left(4 \times 10^{14} \mathrm{~Hz}\right)$ and the X-ray band $(0.3 \mathrm{keV})$, respectively. The dotted lines (Opt- 4 or X-ray-4) represent the flux density of Region 2 before the density jump and the flux density of Region 4 after the jump. The dashed lines (Opt-3 or X-ray-3) are the contribution from Region 3 after the density jump. The dash-dotted lines (Opt-2 or X-ray-2) mark the flux density of Region 2 after the jump. Total flux densities of all the components are presented by the solid lines (Opt-tot or X-ray-tot). For a similar plot, see [47].

leading to the emergence of the optical rebrightening and the X-ray plateau simultaneously. Due to the effect of the equal arrival time surface $[54,55,72]$, the peak time of the optical rebrightening would be delayed, that is, larger than $T_{\text {sd }}$. However, this kind of delay in X-rays can be ignored. As a result, optical rebrightenings are relatively easier to emerge than "X-ray rebrightenings." In other words, it is often the case that only a X-ray plateau or no equivalent feature in Xrays accompanies the optical rebrightening. This property in our model is interestingly consistent with many observations. In Figure 6, optical afterglows are calculated using different values of $B_{\mathrm{NS}}$. From these results, it is found that the flux from the long-lasting RS would account for the shallow optical rebrightening. The $e^{+} e^{-}$wind model has been applied (paper submitted) to explain the afterglows of GRB 080413B [73], GRB 090426 [74], GRB 091029 [75], and GRB 100814A [20].

Since the flux from the RS is sensitive to $L_{w}$ and $T_{\text {sd }}$, the $e^{+} e^{-}$wind scenario provides a useful way to probe the characteristics of newly born magnetars. Considering the fact that $L_{w}$ and $T_{\text {sd }}$ are uniquely determined by the parameter $B_{\mathrm{NS}}$, we suggest that $B_{\mathrm{NS}}$ may be constrained from the fitting to the observed rebrightenings.

\section{Discussion}

In this review, we show that the density jump scenario could not account for many of the observed optical rebrightenings in GRB afterglows. Furthermore, we classify the observed afterglows with optical rebrightenings into two groups and investigate their intrinsic origin. The afterglows with steep optical rebrightenings are interpreted by the delayed energy injection model, which is associated with a central accreting $\mathrm{BH}$. Meanwhile, the afterglows with shallow optical rebrightenings are explained by the $e^{+} e^{-}$wind model, of which the central engine is a magnetar.

Although the above two groups of afterglows have similar origins for rebrightenings, some other factors will make the situation different. The observational data of some afterglows clearly show that their spectral indices are evolving [75]. It is hard to satisfactorily model them only by using the simple models discussed here and/or other customary models. For the simple $e^{+} e^{-}$wind model or the two-component jet model, the spectral evolution would occur only when the characteristic frequencies are crossing the observational band, or when later component begins to dominate, but it cannot work well for some special GRBs. In such cases, including 


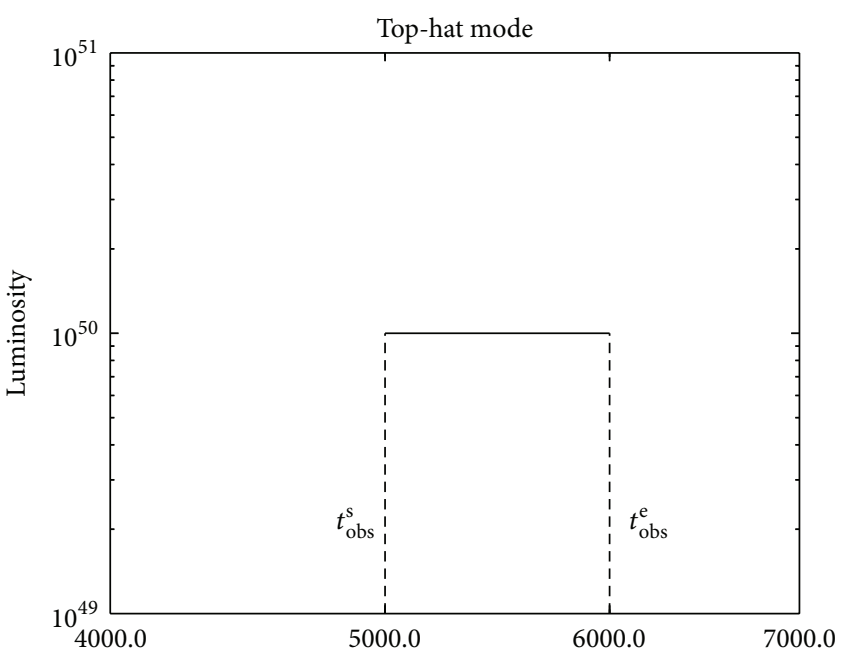

(a)

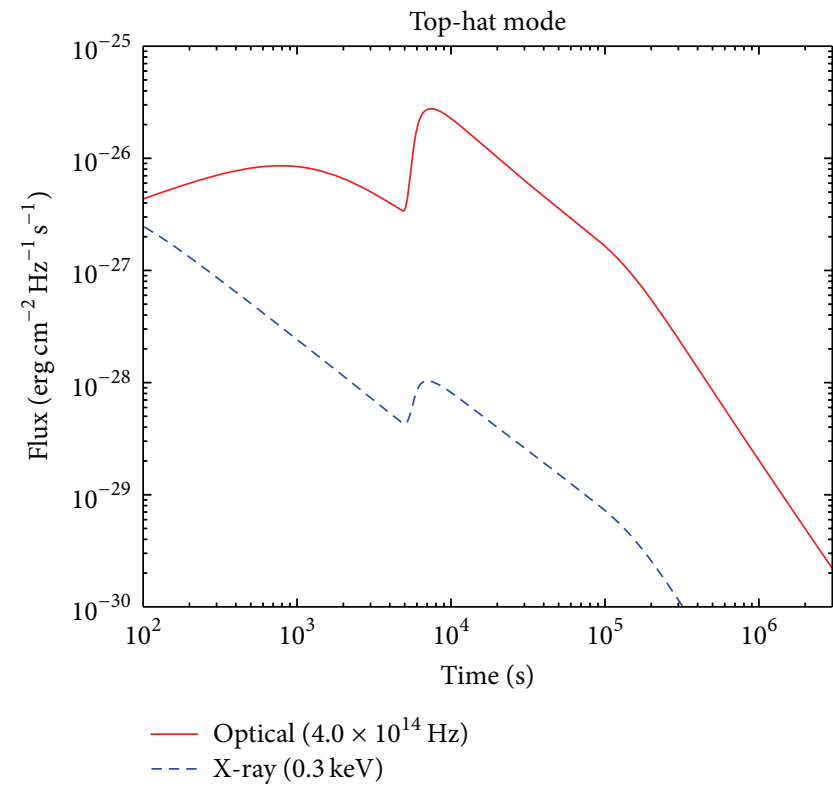

(c)

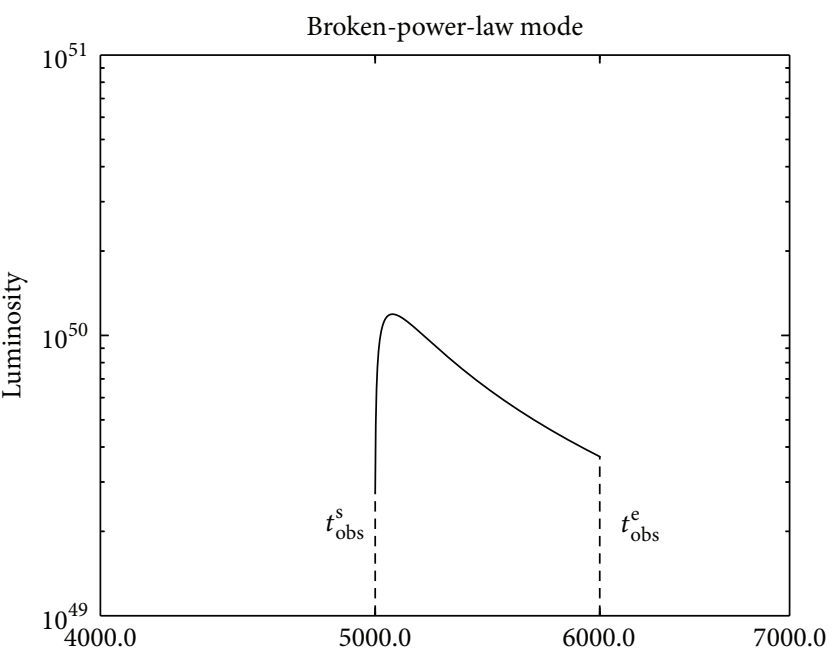

(b)

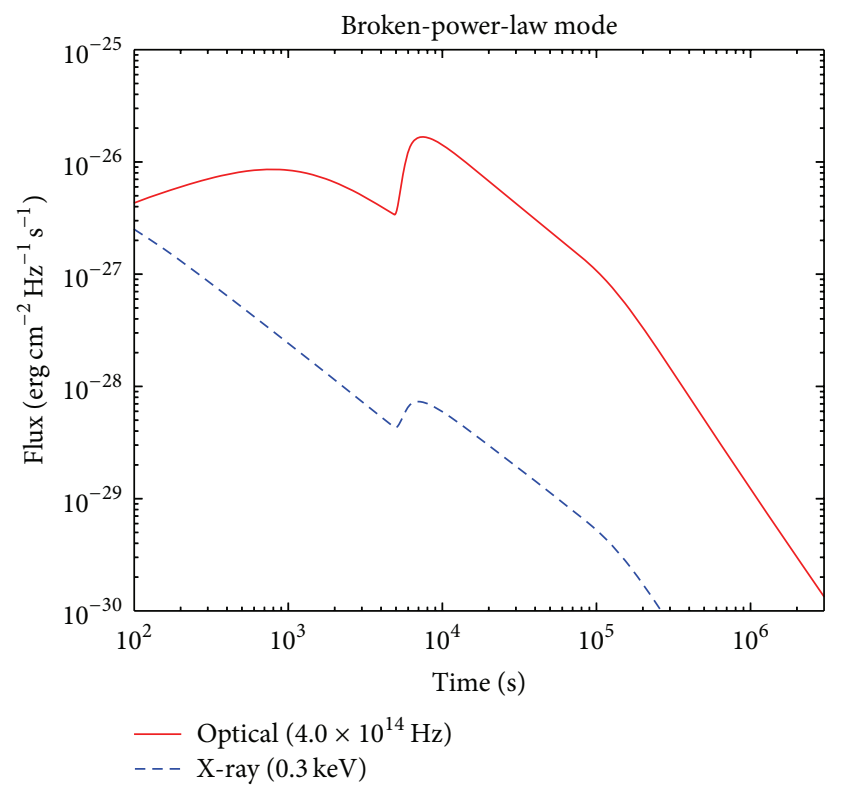

(d)

Figure 3: (a) Schematic illustration of the top-hat injection mode, in which $L_{0}=10^{50} \mathrm{erg} \mathrm{s}^{-1}$ (isotropic), $t_{\mathrm{obs}}^{\mathrm{s}}=5000 \mathrm{~s}$, and $t_{\mathrm{obs}}^{\mathrm{e}}=6000 \mathrm{~s}$. (b) Schematic illustration of the broken-power-law (see (4)) injection mode, in which $L_{\mathrm{p}}=10^{50} \mathrm{erg} \mathrm{s}^{-1}$ (isotropic), $t_{\mathrm{obs}}^{\mathrm{s}}=5000 \mathrm{~s}, t_{\mathrm{obs}}^{\mathrm{p}}=5200 \mathrm{~s}$, $t_{\mathrm{obs}}^{\mathrm{e}}=6000 \mathrm{~s}, \alpha_{r}=0.5, \alpha_{d}=-1.5$, and $\mathrm{s}=0.5$. (c) and (d) show the corresponding afterglows of the two modes correspondingly. In the calculations, a redshift of $z=1$ is assumed. Similar plots can be found at [40].

some microphysical processes may be necessary to match the observations. For example, in the varying microphysical parameters scenarios [36], the varying electron distribution index $p$ would help to explain some unexpected spectral evolutions [75]. Varying microphysical parameters may be related to the acceleration performance of relativistic shocks [76]. Since the acceleration performance of shocks may depend on the magnetization (or other factors) of the plasma and the magnetization is highly variable [77], some special afterglows are foreseeable.

It has been suggested that the two-component jets could also account for some shallow rebrightenings. The collapsar model of long-duration GRBs offers a natural mechanism to generate two-component jets; that is, a high speed jet emerging from a star is accompanied by a relatively slow cocoon $[78,79]$. The rebrightening lightcurve itself will not help to definitely discriminate the $e^{+} e^{-}$wind model from the two-component jet model, since the role of the wide jet is somehow similar to the role of the RS. However, there is another way that can help us in the future. A two-component jet should be associated with a collapsar. If the rebrightening is observed to be associated with the double NS merger (by detections of the gravitational waves [80]) but not a collapsar, then the $e^{+} e^{-}$wind model would be preferred.

The intrinsic origins of optical rebrightenings would help to probe the characteristics of central engines. In the delayed 


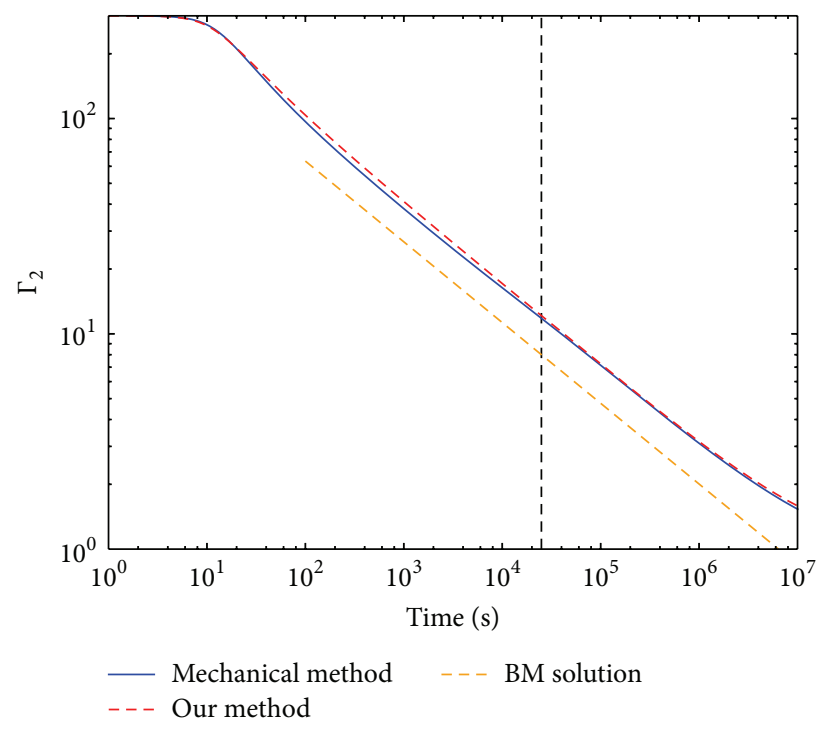

Figure 4: Comparison of the two methods used in solving the temporal evolution of $\Gamma_{2}$ in the $e^{+} e^{-}$wind model. The initial parameter values are $E_{K \text {,iso }}=8.0 \times 10^{52} \mathrm{erg}, \Gamma_{2,0}=150, n_{0}=0.1 \mathrm{~cm}^{-3}$, $\Gamma_{4}=10^{4}$, and $B_{\mathrm{NS}}=2 \times 10^{14} \mathrm{G}$. The thick dashed orange line represents the $\mathrm{BM}$ solution (schematic); that is, $\Gamma \propto t_{\mathrm{obs}}^{3 / 8}$ and the vertical dashed line denote the position of $T_{\mathrm{sd}}$.

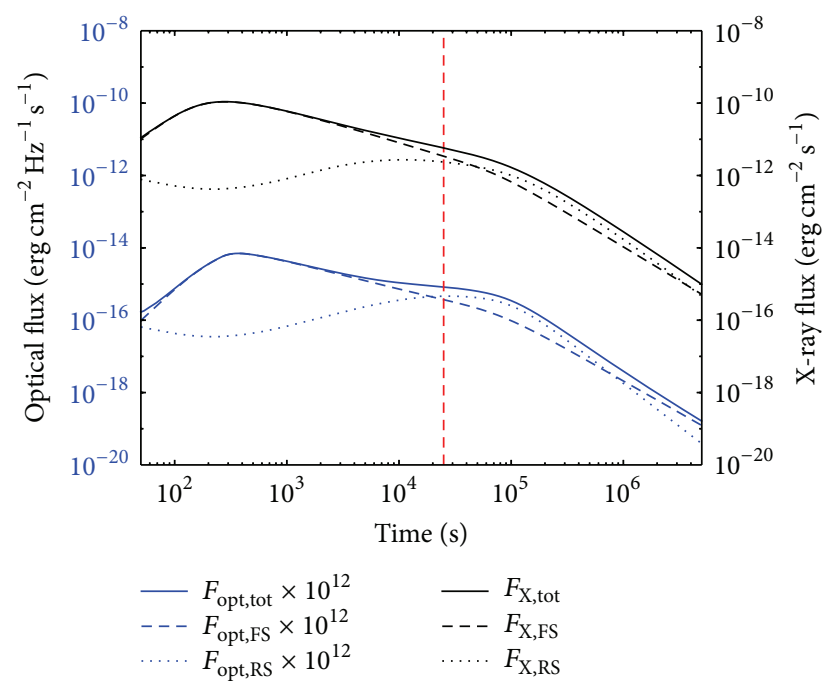

FIgURE 5: Corresponding afterglow lightcurves of Figure 4. The dashed lines represent the flux from Region 3, while the dotted lines are emissions from Region 2. The total flux is shown as the solid lines. In the calculations, the optical band is taken as $4.0 \times 10^{14} \mathrm{~Hz}$ and the $\mathrm{X}$-ray band is taken as $0.3-10 \mathrm{keV}$. The red dashed vertical line marks the position of $T_{\text {sd }}$.

energy injection model, $t_{\mathrm{fb}}$ can be derived from the start time of the rebrightening, and $r_{\mathrm{fb}}$ can thus be obtained. If the observational data is good enough, $L$ can be constrained in the fitting process, and we can estimate the total mass of the fall-back material. Furthermore, the fall-back accretion theory requires that the steep optical rebrightening should be

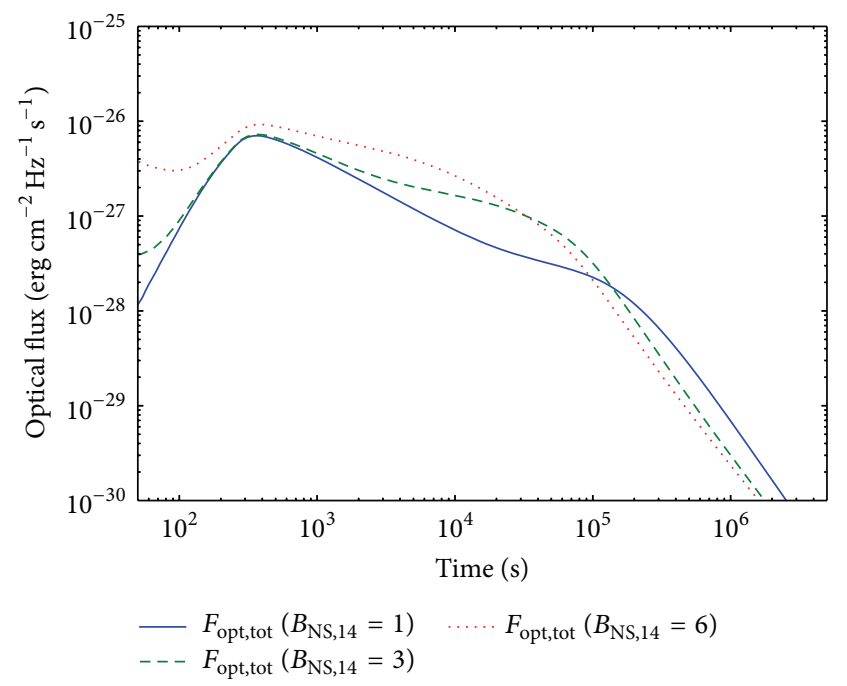

Figure 6: Optical lightcurves in the electron-positron wind model with different $B_{\mathrm{NS}}$. All the parameters are the same as those in Figure 5, except for $B_{\mathrm{NS}, 14}=1$ (solid line), $B_{\mathrm{NS}, 14}=3$ (dashed line), and $B_{\mathrm{NS}, 14}=6$ (dotted line).

accompanied by a low energy supernova, in which the fallback material can survive during the explosion. This model thus can be tested by future observations of GRB-supernova association. In the $e^{+} e^{-}$wind model, $T_{\text {sd }}$ can be roughly inferred from the peak time of the optical rebrightening. In general, an earlier rebrightening means that $B_{\mathrm{NS}}$ is larger or $P_{\mathrm{NS}}$ is smaller. So $B_{\mathrm{NS}}$ and $P_{\mathrm{NS}}$ can be constrained from observations. Thus the $e^{+} e^{-}$model provides a potential way to probe the characteristics of the central magnetar.

\section{Competing Interests}

The authors declare that there are no competing interests related to this paper.

\section{Acknowledgments}

The authors thank Liang Li for helpful discussion. This work was supported by the National Basic Research Program of China with Grant no. 2014 CB845800 and by the National Natural Science Foundation of China with Grant no. 11473012 .

\section{References}

[1] S. E. Woosley, "Gamma-ray bursts from stellar mass accretion disks around black holes," The Astrophysical Journal, vol. 405, no. 1, pp. 273-277, 1993.

[2] R. Popham, S. E. Woosley, and C. Fryer, "Hyperaccreting black holes and gamma-ray bursts," The Astrophysical Journal, vol. 518, no. 1, pp. 356-374, 1999.

[3] A. I. Macfadyen, S. E. Woosley, and A. Heger, "Supernovae, jets, and collapsars," The Astrophysical Journal, vol. 550, no. 1, pp. 410-425, 2001. 
[4] R. Narayan, T. Piran, and P. Kumar, "Accretion models of gamma-ray bursts," The Astrophysical Journal, vol. 557, no. 2, pp. 949-957, 2001.

[5] D. Eichler, M. Livio, T. Piran, and D. N. Schramm, "Nucleosynthesis, neutrino bursts and $\gamma$-rays from coalescing neutron stars," Nature, vol. 340, no. 6229, pp. 126-128, 1989.

[6] B. Paczynski, "Cosmological gamma-ray bursts," Acta Astronomica, vol. 41, no. 4, pp. 257-267, 1991.

[7] J. Grindlay, S. Portegies Zwart, and S. McMillan, "Short gammaray bursts from binary neutron star mergers in globular clusters," Nature Physics, vol. 2, no. 2, pp. 116-119, 2006.

[8] R. D. Blandford and C. F. McKee, "Fluid dynamics of relativistic blast waves," Physics of Fluids, vol. 19, no. 8, pp. 1130-1138, 1976.

[9] T. Piran, A. Shemi, and R. Narayan, "Hydrodynamics of relativistic fireballs," Monthly Notices of the Royal Astronomical Society, vol. 263, no. 4, pp. 861-867, 1993.

[10] P. Mészáros and M. J. Rees, "Optical and long-wavelength afterglow from gamma-ray bursts," The Astrophysical Journal, vol. 476, no. 1, pp. 232-237, 1997.

[11] R. Sari and T. Piran, "Predictions for the very early afterglow and the optical flash," The Astrophysical Journal, vol. 520, no. 2, pp. 641-649, 1999.

[12] H. Gao, W.-H. Lei, Y.-C. Zou, X.-F. Wu, and B. Zhang, "A complete reference of the analytical synchrotron external shock models of gamma-ray bursts," New Astronomy Reviews, vol. 57, no. 6, pp. 141-190, 2013.

[13] N. Gehrels, G. Chincarini, P. Giommi et al., "The swift gammaray burst mission," The Astrophysical Journal, vol. 611, no. 2, pp. 1005-1020, 2004.

[14] D. N. Burrows, J. E. Hill, J. A. Nousek et al., "The swift X-ray telescope," Space Science Reviews, vol. 120, no. 3-4, pp. 165-195, 2005.

[15] N. Gehrels, E. Ramirez-Ruiz, and D. B. Fox, "Gamma-ray bursts in the Swift era," Annual Review of Astronomy and Astrophysics, vol. 47, no. 1, pp. 567-617, 2009.

[16] J. Greiner, W. Bornemann, C. Clemens et al., "GROND-a 7channel imager," Publications of the Astronomical Society of the Pacific, vol. 120, no. 866, pp. 405-424, 2008.

[17] B. Zhang, Y. Z. Fan, J. Dyks et al., "Physical processes shaping gamma-ray burst X-ray afterglow light curves: theoretical implications from the swift X-ray telescope observations," The Astrophysical Journal, vol. 642, no. 1, pp. 354-370, 2006.

[18] A. Melandri, F. J. Virgili, C. Guidorzi et al., "The nature of the late achromatic bump in GRB 120326A," Astronomy and Astrophysics, vol. 572, p. A55, 2014.

[19] S. J. Hou, J. J. Geng, K. Wang et al., "The origin of the plateau and late rebrightening in the afterglow of GRB 120326A," The Astrophysical Journal, vol. 785, no. 2, p. 113, 2014.

[20] M. De Pasquale, N. P. M. Kuin, S. Oates et al., “The optical rebrightening of GRB100814A: an interplay of forward and reverse shocks?" Monthly Notices of the Royal Astronomical Society, vol. 449, no. 1, pp. 1024-1042, 2015.

[21] H. Gao and P. Mészáros, "Reverse shock emission in gammaray bursts revisited," Advances in Astronomy, vol. 2015, Article ID 192383, 16 pages, 2015.

[22] P. Kumar and B. Zhang, "The physics of gamma-ray bursts \& relativistic jets," Physics Reports, vol. 561, pp. 1-109, 2015.

[23] L. Li, X.-F. Wu, Y.-F. Huang et al., "A correlated study of optical and X-ray afterglows of GRBs," The Astrophysical Journal, vol. 805, no. 1, article 13, 2015.
[24] Z. G. Dai and T. Lu, "Gamma-ray burst afterglows and evolution of postburst fireballs with energy injection from strongly magnetic millisecond pulsars," Astronomy and Astrophysics, vol. 333, no. 3, pp. L87-L90, 1998.

[25] B. Zhang and P. Mészáros, "Gamma-ray burst afterglow with continuous energy injection: signature of a highly magnetized millisecond pulsar," Astrophysical Journal Letters, vol. 552, no. 1, pp. L35-L38, 2001.

[26] Y.-Z. Fan and D. Xu, "The X-ray afterglow flat segment in short GRB 051221A: energy injection from a millisecond magnetar?" Monthly Notices of the Royal Astronomical Society, vol. 372, no. 1, pp. L19-L22, 2006.

[27] S. Kong and Y. Huang, "Afterglow from GRB 070610/Swift J195509.6+261406: an explanation using the fireball model," Science China Physics, Mechanics, and Astronomy, vol. 53, supplement 1, pp. 94-97, 2010.

[28] B. Zhang and P. Mészáros, "Gamma-ray bursts with continuous energy injection and their afterglow signature," The Astrophysical Journal, vol. 566, no. 2, pp. 712-722, 2002.

[29] Z. G. Dai, "Relativistic wind bubbles and afterglow signatures," The Astrophysical Journal, vol. 606, no. 2, pp. 1000-1005, 2004.

[30] Y. W. Yu, X. W. Liu, and Z. G. Dai, “Observational signatures of high-energy emission during the shallow decay phase of gamma-ray burst X-ray afterglows," The Astrophysical Journal, vol. 671, no. 1, pp. 637-644, 2007.

[31] D. Lazzati, E. Rossi, S. Covino, G. Ghisellini, and D. Malesani, "The afterglow of GRB 021004: surfing on density waves," Astronomy \& Astrophysics, vol. 396, no. 2, pp. L5-L9, 2002.

[32] Z. G. Dai and X. F. Wu, "GRB 030226 in a density-jump medium," Astrophysical Journal Letters, vol. 591, no. 1, pp. L21L24, 2003.

[33] E. Nakar and T. Piran, "Modeling fluctuations in gamma-ray burst afterglow light curves," The Astrophysical Journal, vol. 598, no. 1, pp. 400-410, 2003.

[34] E. Berger, S. R. Kulkarni, G. Pooley et al., "A common origin for cosmic explosions inferred from calorimetry of GRB 030329," Nature, vol. 426, no. 6963, pp. 154-157, 2003.

[35] Y. F. Huang, X. F. Wu, Z. G. Dai, H. T. Ma, and T. Lu, "Rebrightening of XRF 030723: further evidence for a twocomponent jet in a gamma-ray burst," The Astrophysical Journal, vol. 605, no. 1, pp. 300-306, 2004.

[36] S. W. Kong, A. Y. L. Wong, Y. F. Huang, and K. S. Cheng, "Variation of microphysics in wind bubbles: an alternative mechanism for explaining the rebrightenings in gamma-ray burst afterglows," Monthly Notices of the Royal Astronomical Society, vol. 402, no. 1, pp. 409-416, 2010.

[37] R. Perna, P. J. Armitage, and B. Zhang, "Flares in long and short gamma-ray bursts: a common origin in a hyperaccreting accretion disk," Astrophysical Journal Letters, vol. 636, no. 1, pp. L29-L32, 2006.

[38] P. Kumar, R. Narayan, and J. L. Johnson, "Mass fall-back and accretion in the central engine of gamma-ray bursts," Monthly Notices of the Royal Astronomical Society, vol. 388, no. 4, pp. $1729-1742,2008$.

[39] X.-F. Wu, S.-J. Hou, and W.-H. Lei, "Giant X-ray bump in GRB 121027A: evidence for fall-back disk accretion," Astrophysical Journal Letters, vol. 767, no. 2, article L36, 2013.

[40] J. J. Geng, X. F. Wu, Y. F. Huang, and Y. B. Yu, "Delayed energy injection model for gamma-ray burst afterglows," The Astrophysical Journal, vol. 779, no. 1, article 28, 2013. 
[41] Y. W. Yu and Z. G. Dai, "Shallow decay phase of GRB Xray afterglows from relativistic wind bubbles," Astronomy and Astrophysics, vol. 470, no. 1, pp. 119-122, 2007.

[42] H. J. van Eerten, Z. Meliani, R. A. M. J. Wijers, and R. Keppens, "No visible optical variability from a relativistic blast wave encountering a wind termination shock," Monthly Notices of the Royal Astronomical Society, vol. 398, no. 1, pp. L63-L67, 2009.

[43] I. Gat, H. van Eerten, and A. MacFadyen, "No flares from gamma-ray burst afterglow blast waves encountering sudden circumburst density change," The Astrophysical Journal, vol. 773, no. 1, article 2, 2013.

[44] Y. F. Huang, Z. G. Dai, and T. Lu, "A generic dynamical model of gamma-ray burst remnants," Monthly Notices of the Royal Astronomical Society, vol. 309, no. 2, pp. 513-516, 1999.

[45] Y. F. Huang, L. J. Gou, Z. G. Dai, and T. Lu, "Overall evolution of jetted gamma-ray burst ejecta," The Astrophysical Journal, vol. 543, no. 1, pp. 90-96, 2000.

[46] A. Pe'er, "Dynamical model of an expanding shell," Astrophysical Journal Letters, vol. 752, no. 1, article L8, 2012.

[47] J. J. Geng, X. F. Wu, L. Li, Y. F. Huang, and Z. G. Dai, "Revisiting the emission from relativistic blast waves in a density-jump medium," The Astrophysical Journal, vol. 792, no. 1, article 31, 2014.

[48] R. Sari and T. Piran, "Hydrodynamic timescales and temporal structure of gamma-ray bursts," Astrophysical Journal Letters, vol. 455, p. L143, 1995.

[49] S. Kobayashi, T. Piran, and R. Sari, "Hydrodynamics of a relativistic fireball: the complete evolution," The Astrophysical Journal, vol. 513, no. 2, pp. 669-678, 1999.

[50] R. Sari, T. Piran, and R. Narayan, "Spectra and light curves of gamma-ray burst afterglows," The Astrophysical Journal Letters, vol. 497, no. 1, pp. L17-L20, 1998.

[51] G. B. Rybicki and A. P. Lightman, Radiative Processes in Astrophysics, Wiley-Interscience, New York, NY, USA, 1979.

[52] Y. Fan and T. Piran, "Gamma-ray burst efficiency and possible physical processes shaping the early afterglow," Monthly Notices of the Royal Astronomical Society, vol. 369, no. 1, pp. 197-206, 2006.

[53] X.-Y. Wang, H.-N. He, Z. Li, X.-F. Wu, and Z.-G. Dai, "Klein-nishina effects on the high-energy afterglow emission of gamma-ray bursts," The Astrophysical Journal, vol. 712, no. 2, pp. 1232-1240, 2010.

[54] E. Waxman, "Angular size and emission timescales of relativistic fireballs," The Astrophysical Journal Letters, vol. 491, no. 1, pp. L19-L22, 1997.

[55] J. Granot, T. Piran, and R. Sari, "Images and spectra from the interior of a relativistic fireball," The Astrophysical Journal, vol. 513, no. 2, pp. 679-689, 1999.

[56] Y. F. Huang, Z. G. Dai, and T. Lu, "On the optical light curves of afterglows from jetted gamma-ray burst ejecta: effects of parameters," Monthly Notices of the Royal Astronomical Society, vol. 316, no. 4, pp. 943-949, 2000.

[57] Z. G. Dai and T. Lu, "Hydrodynamics of relativistic blast waves in a density-jump medium and their emission signature," The Astrophysical Journal Letters, vol. 565, no. 2, pp. L87-L90, 2002.

[58] R. D. Blandford and R. L. Znajek, "Electromagnetic extraction of energy from Kerr black holes," Monthly Notices of the Royal Astronomical Society, vol. 179, no. 3, pp. 433-456, 1977.

[59] H. K. Lee, R. A. M. J. Wijers, and G. E. Brown, "The BlandfordZnajek process as a central engine for a gamma-ray burst," Physics Reports, vol. 325, no. 3, pp. 83-114, 2000.
[60] F. Yuan and B. Zhang, "Episodic jets as the central engine of gamma-ray bursts," The Astrophysical Journal, vol. 757, no. 1, article 56, 2012.

[61] W. Zhang, S. E. Woosley, and A. Heger, "Fallback and black hole production in massive stars," The Astrophysical Journal, vol. 679, no. 1, pp. 639-654, 2008.

[62] Z. G. Dai and R.-Y. Liu, "Spin evolution of millisecond magnetars with hyperaccreting fallback disks: implications for early afterglows of gamma-ray bursts," The Astrophysical Journal, vol. 759, no. 1, article 58, 2012.

[63] M. Nardini, J. Greiner, T. Krühler et al., "On the nature of the extremely fast optical rebrightening of the afterglow of GRB 081029," Astronomy and Astrophysics, vol. 531, p. A39, 2011.

[64] S. T. Holland, M. De Pasquale, J. Mao et al., "GRB 081029: a gamma-ray burst with a multi-component afterglow," The Astrophysical Journal, vol. 745, no. 1, article 41, 2012.

[65] J. Greiner, T. Krühler, M. Nardini et al., "The unusual afterglow of the gamma-ray burst 100621A," Astronomy and Astrophysics, vol. 560, article A70, 2013.

[66] M. J. Rees and J. E. Gunn, "The origin of the magnetic field and relativistic particles in the Crab Nebula," Monthly Notices of the Royal Astronomical Society, vol. 167, no. 1, pp. 1-12, 1974.

[67] Y. Lyubarsky and J. G. Kirk, "Reconnection in a striped pulsar wind," The Astrophysical Journal, vol. 547, no. 1, pp. 437-448, 2001.

[68] B. D. Metzger and A. L. Piro, "Optical and X-ray emission from stable millisecond magnetars formed from the merger of binary neutron stars," Monthly Notices of the Royal Astronomical Society, vol. 439, no. 4, pp. 3916-3930, 2014.

[69] S. L. Shapiro and S. A. Teukolsky, Black Holes, White Dwarfs and Neutron Stars: The Physics of Compact Objects, National Science Foundation, Arlington, Va, USA; Wiley-Interscience, New York, NY, USA, 1983.

[70] A. M. Beloborodov and Z. L. Uhm, "Mechanical model for relativistic blast waves," Astro-Physical Journal Letters, vol. 651, no. 2, pp. L1-L4, 2006.

[71] Z. L. Uhm, "A semi-analytic formulation for relativistic blast waves with a long-lived reverse shock," The Astrophysical Journal, vol. 733, no. 2, article 86, 2011.

[72] Y.-F. Huang, Y. Lu, A. Y. L. Wong, and K. S. Cheng, "A detailed study on the equal arrival time surface effect in gamma-ray burst afterglows," Chinese Journal of Astronomy and Astrophysics, vol. 7, no. 3, pp. 397-404, 2007.

[73] R. Filgas, T. Krühler, J. Greiner et al., "The two-component jet of GRB 080413B," Astronomy and Astrophysics, vol. 526, article A113, 2011.

[74] A. Nicuesa Guelbenzu, S. Klose, A. Rossi et al., "GRB 090426: discovery of a jet break in a short burst afterglow," Astronomy and Astrophysics, vol. 531, article L6, 2011.

[75] R. Filgas, J. Greiner, P. Schady et al., "GRB 091029: at the limit of the fireball scenario," Astronomy and Astrophysics, vol. 546, article A101, 2012.

[76] L. Sironi, A. Spitkovsky, and J. Arons, "The maximum energy of accelerated particles in relativistic collisionless shocks," The Astrophysical Journal, vol. 771, no. 1, article 54, 2013.

[77] B. Zhang and S. Kobayashi, "Gamma-ray burst early afterglows: reverse shock emission from an arbitrarily magnetized ejecta," The Astrophysical Journal, vol. 628, no. 1, pp. 315-334, 2005.

[78] E. Ramirez-Ruiz, A. Celotti, and M. J. Rees, "Events in the life of a cocoon surrounding a light, collapsar jet," Monthly Notices of the Royal Astronomical Society, vol. 337, no. 4, pp. 1349-1356, 2002 . 
[79] D. Lazzati, B. J. Morsony, and D. López-Cámara, "Numerical simulations of gamma-ray burst explosions," Journal of High Energy Astrophysics, vol. 7, pp. 17-22, 2015.

[80] B. P. Abbott, R. Abbott, T. D. Abbott et al., "Observation of gravitational waves from a binary black hole merger," Physical Review Letters, vol. 116, no. 6, Article ID 061102, 2016. 

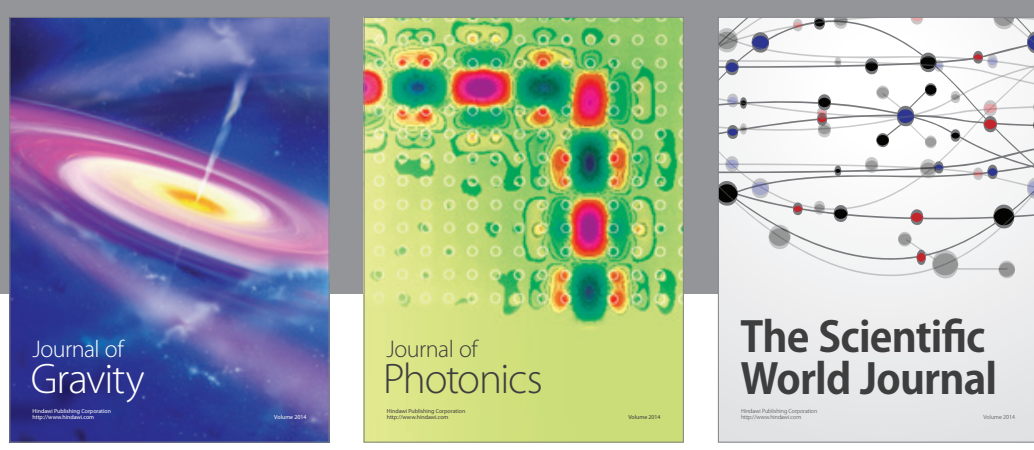

The Scientific World Journal
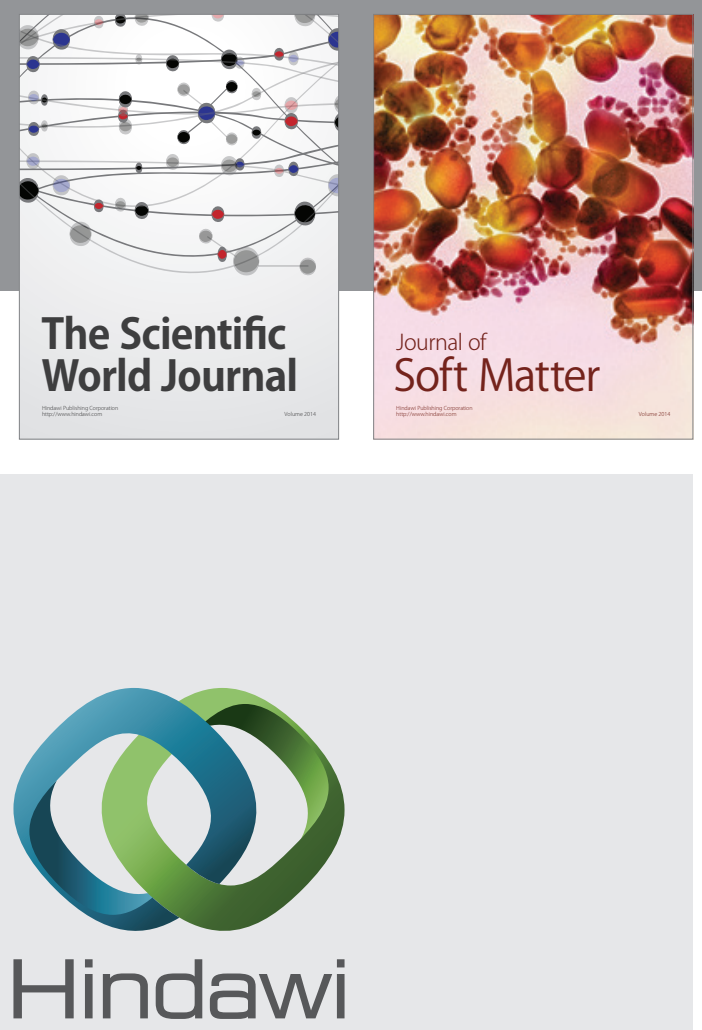

Submit your manuscripts at

http://www.hindawi.com

nternational Journal of

Statistical Mechanics
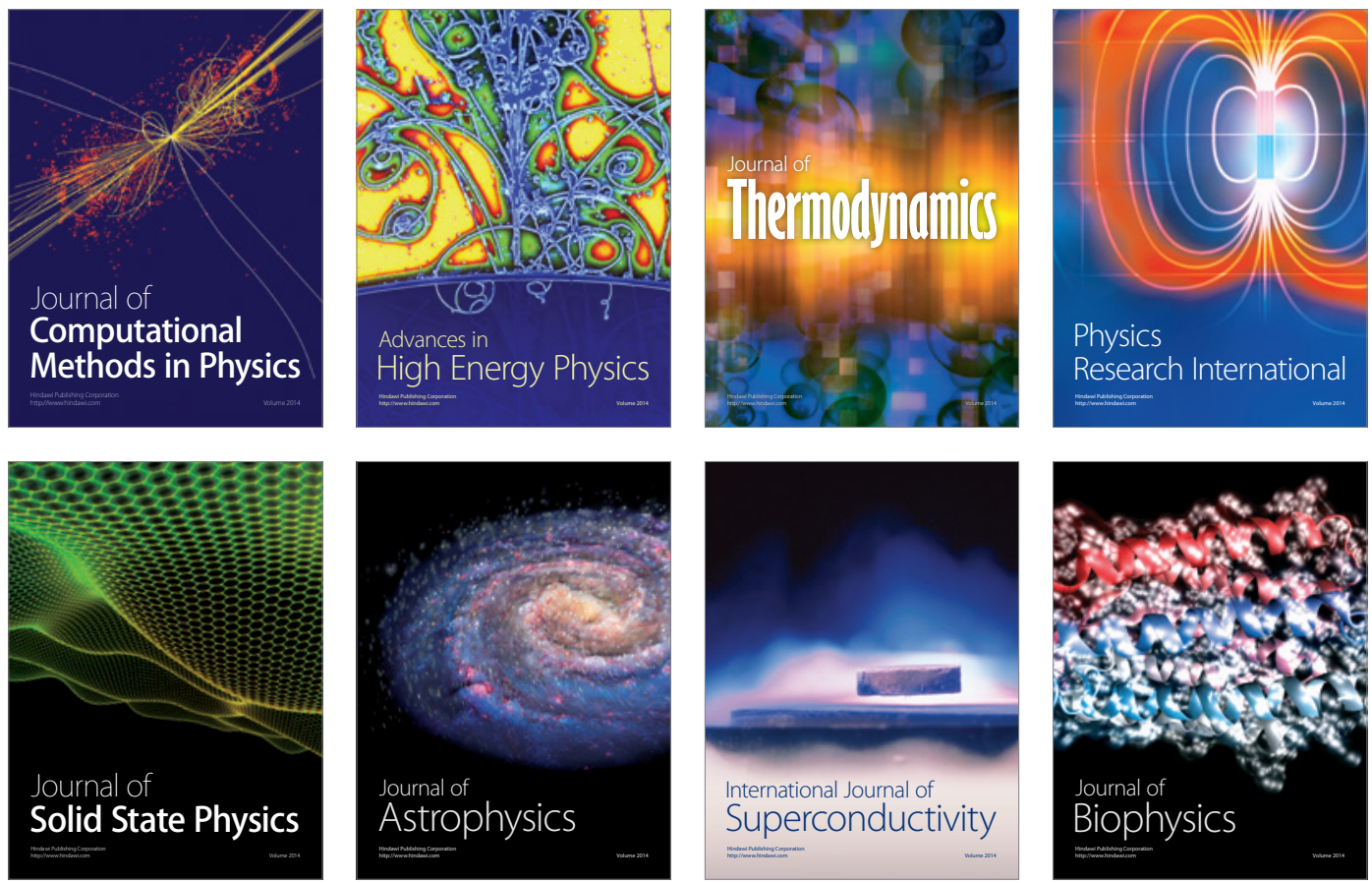
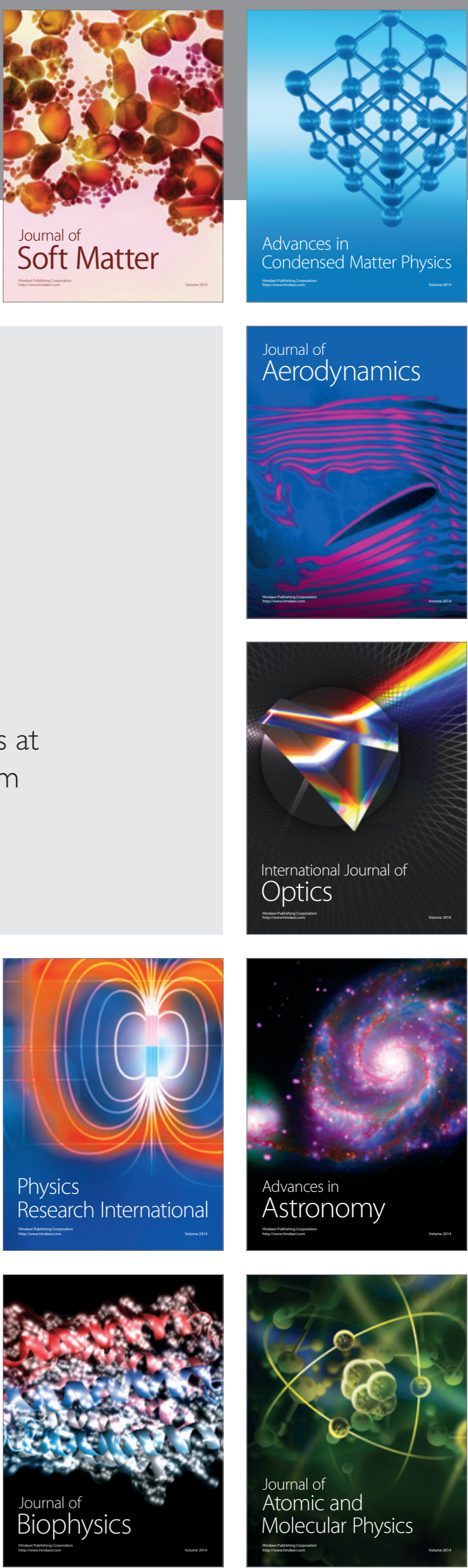